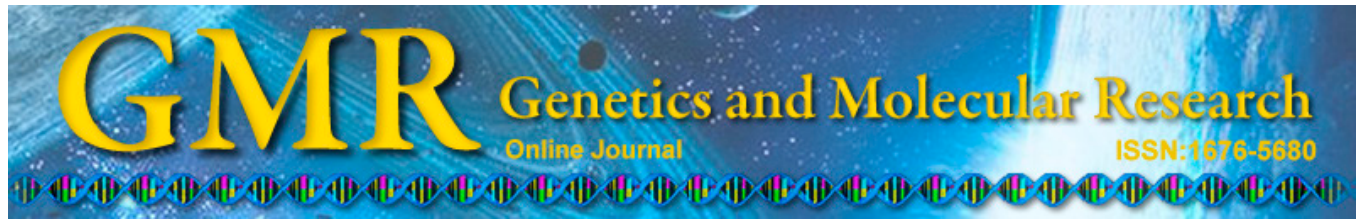

\title{
Effects of silicon on seed setting rate of rice intersubspecific hybrids
}

\author{
W.-C. Li ${ }^{1}$, L. Zhang ${ }^{1}$, J. Wang ${ }^{1}$, D. Wang', T.-X. Wang ${ }^{1}$ and C.-X. Duan ${ }^{2}$ \\ ${ }^{1}$ College of Life Sciences, Henan Normal University, Xinxiang, China \\ ${ }^{2}$ National Key Facility for Crop Gene Resources and Genetic Improvement, \\ Institute of Crop Science, Chinese Academy of Agricultural Sciences, \\ Beijing, China \\ Corresponding author: C.-X. Duan \\ E-mail: duancx_1wc@163.com
}

Genet. Mol. Res. 14 (3): 10359-10364 (2015)

Received January 21, 2015

Accepted June 24, 2015

Published September 1, 2015

DOI http://dx.doi.org/10.4238/2015.September.1.2

\begin{abstract}
The present study found semi-sterility in rice intersubspecific hybrids of 'Taichung 65' $x$ 'Guangluai 4' and 'Ludao' $\mathrm{x}$ 'Qiuguang'. Embryo sac fertility was evaluated using the overall staining transparent method. The results showed that the embryo sac contained a normal egg cell, normal synergid cells, polar nuclei cells, and antipodal cells, indicating that semi-sterility was caused mainly by pollen semi-sterility. In the pot experiment, the effects of silicon on the seed-setting rate of the two intersubspecific hybrids were examined. The results showed that the rate of anther dehiscence, number of pollen per stigma of $\mathrm{Fl}$ plants, potential of pollen grain germination, and fertility of the spikelet were significantly improved by the utilization of silicon fertilizer.
\end{abstract}

Key words: Embryo sac fertility; Intersubspecific hybrid; Silicon; Seed setting rate; Semi-sterility 


\section{INTRODUCTION}

The F1 hybrid between indica and japonica varieties of rice shows strong heterosis, but the use of heterosis is limited by its ubiquitous semi-sterility. Hybrid semi-sterility can be manifested as female infertility, male sterility, anther-unslit, and dichogamy (Liu et al., 1997).

In hybrids with male semi-infertility and normal pistils, pollen fertility is approximately $50 \%$. Theoretically, a small amount of pollen is required for fertilization, but the spikelet is semiinfertile. Kitamura (1962) first reported that anther-unslit decreased the seed-setting rate of F1 hybrid rice. Ekanayake et al. (1990) found that at least 20 pollens per stigma are required to ensure normal fertilization; otherwise, the flowers will be infertile. In addition, rice is a typical siliconphile plant. Previous experiments showed that the plant had the greatest demand for silicon during the reproductive growth phase, and the number of fertile florets after the utilization of silicon fertilizer during the floret-phase was 1.5-fold higher than that in the control group (Ma et al., 1989). A large number of field experiments demonstrated that application of silicon fertilizer could increase rice yield by 10-26\% (Epstein, 1999; Shang et al., 2009). However, whether the application of silicon can improve the seed-setting rate of F1 between rice subspecies has not been reported previously. In this study, we examined the impact of silicon on pollen dehiscence, pollen viability, and seed-setting rate of spikelets in an intersubspecific F1 hybrid to provide a theoretical basis for the silicon-enhancement effect on the seed-setting rates of intersubspecific F1.

\section{MATERIAL AND METHODS}

\section{Plant material and process}

Japonica ('Taichung 65' and 'Guangluai 4') and indica ('Ludao' and 'Qiuguang') varieties of rice were used in this study. The hybrids used were the F1 generation of 'Taichung 65' x 'Guangluai 4' and 'Ludao' x 'Qiuguang'.

In rice growth masasue, seedlings were washed with quartz sand at $30^{\circ} \mathrm{C}$ for 14 days before being transplanted in a 2-L porcelain culture tank. For both the control group (without silicon) and silicon group, a porcelain culture tank was lined with plastic wrap to prevent the container from interacting with the silicon in the nutrient solutions. The nutrient solution was prepared based on the formulation of the International Rice Institute (Yoshida et al., 1976). Silicon was added in the form of silicic acid at a concentration of $1.5 \mathrm{mM}$. Silicic acid was exchanged from sodium styrene by using a hyperacidity cinnamic cationic switching resin (Shanghai Chemical Reagent Company of China Pharmaceutical Group, Shanghai, China). A month after transplantation, the rice was treated with silicon or remained untreated, and the plants began heading during the 4 th month.

\section{Methods}

The pollen fertility survey was conducted as described by Li et al. (2007). Soon before plant blossoming, 3 glumous flowers were removed from the main spike of each plant and placed in a solution of ethanol:acetic acid $(3: 1)$, which was stored at $4^{\circ} \mathrm{C}$. The samples were stained with $1 \% \mathrm{I}_{2}-\mathrm{KI}$ and observed under a $10 \mathrm{X}$ (10-fold) microscope, and the fertility was determined based on pollen form and color. For the spikelet fertility survey, at the mature stage, 3 spikes per plant were collected to survey spike fertility, and the average value was calculated as spikelet fertility per plant. 
Embryo sac fertility was examined using the Ehrlich hematoxylin overall stainingtransparent method, and samples of flowering F1 of 'Taichung 65' x 'Guangluai 4' and 'Ludao' x 'Qiuguang' were selected. Samples were collected from a mature embryo sac. A total of 300 embryo sacs from different parts of different spikelets were randomly selected and fixed in the formalin-acetic acid-alcohol fixative for $24 \mathrm{~h}$. After rehydration, the samples were stained by Ehrlich hematoxylin overall staining with love's hematoxylin stain for 20-40 min, and then washed with distilled water for 2 to 3 times $(24 \mathrm{~h}$ ), followed by washing with water 3 to 4 times ( 1 to 2 days) until the color of materials changed from purple to gray-blue. The samples were dehydrated with ethanol before treatment with methyl salicylate to make the tissues transparent. After 24 $\mathrm{h}$, the samples were observed under the Olympus BH-2 microscope (Olympus, Tokyo, Japan).

To measure pollen on the stigma, 10 opened flowers were collected and fixed in formalin-acetic acid-alcohol fixative, and then stained with aniline blue. The number of pollens on the stigma was observed by fluorescence microscopy (Matsui et al., 2000).

Pollen germination in vitro was performed using the method described by Wang et al. (2000), with some modifications. Pollen germination in the control and treated groups in vitro was observed using liquid medium. Pollen germination was observed under a microscope, with pollen tube length greater than or equal to half the diameter of pollen was defined as germination. The experiment was repeated 5 times, examining at least 500 pollens each time.

\section{RESULTS}

\section{Fertility observation}

We examined pollen fertility, spikelet fertility, and embryo sac fertility of the parents ('Taichung 65', 'Guangluai 4', 'Ludao', and 'Qiuguang') and F1 hybrids ('Taichung 65' x 'Guangluai 4' and 'Ludao' x 'Qiuguang') in the control group (without silicon treatment). The fertility of the 4 parents was normal (Figure 1B); pollen and spikelet fertility of 'Taichung 65' x 'Guangluai 4' were 40.1 and $25.8 \%$ respectively, and that of 'Ludao' $x$ 'Qiuguang' were 50.3 (Figure 1A) and 40.3\%, respectively. The embryo sacs of the $2 \mathrm{~F} 1$ hybrids were observed. The normal embryo sac contained 1 egg apparatus (including 1 egg cell and 2 synergid cells), 1 central cell consisting of 2 nuclei (polar nuclei), and antipodal cells (Figure 2A), while the abortive embryo sac only had traces of cell degeneration (Figure 2B) in the normal embryo sac location. A total of 150 embryo sacs were observed in the 2 hybrids. The rates of embryo sac abortion were 5.33\% in 'Taichung 65' x 'Guangluai 4' (8) and 3.33\% 'Ludao' x 'Qiuguang' (5). Therefore, the semi-sterility of intersubspecific F1 hybrids was caused by male semi-sterility.
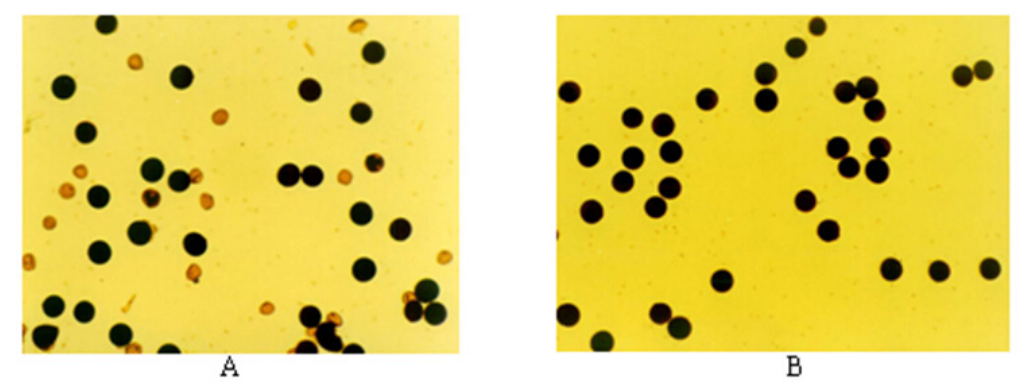

Figure 1. Pollen grain fertility. A. Semi-sterility pollen. B. Fertility pollen. 


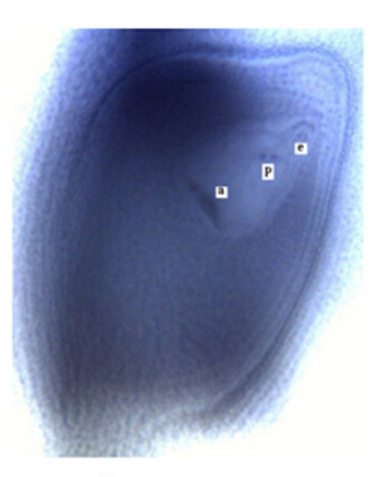

A

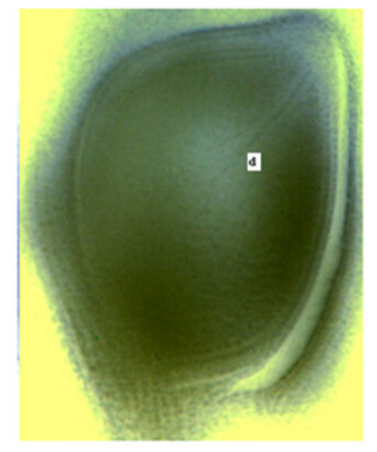

Figure 2. Embryo sac observation using whole stain-clearing technique. A. Fertile embryo sac of Nipponbare with visible antipodal cells (a), polar nucleus (p), and egg organ (e). B. Sterile embryo sac with degenerated cells (d).

\section{Effects of silicon on the number of pollen on the stigma}

Rice seeding rate depends on the fertilization rate, while normal fertilization primarily depends on the number of pollen grains on the stigma. A low number of pollen grains cannot properly fertilize and seed, and pollen germination on the stigma can also affect the setting rate of the spikelet. Pollen fertility and spikelet fertility of the 4 parents were normal, but values for the F1 hybrids were abnorma1 between indica and japonica rice. Thus, only the F1 hybrids were studied.

After flowering for $1 \mathrm{~h}$, the number of pollens scattered on the stigma and the number of pollens germinated in the $2 \mathrm{~F} 1$ hybrids were observed under natural conditions. Two hundred florets of the $2 \mathrm{~F} 1$ hybrids of the silicon group and control group were observed under a fluorescence microscope. Statistical analysis showed that the stigma of the 2 F1 hybrids in the silicon group was similar and could normally accept pollen, with a larger number of pollen grains on the stigma. About $90 \%$ of florets had a pollen number greater than 25 per stigma (Figure 3A), with at least 16 pollen per stigma, while the control group showed a lower number of pollen on the stigma. Approximately $8 \%$ of the florets had more than 20 pollen per stigma, whereas $75 \%$ florets had lesser than 20 pollen per stigma (Figure 3B), and 17\% of florets did not have any pollen on the stigma (Figure 3C).

Figure 3 shows a higher number of pollens germinated on the stigma of F1 hybrids, and there was a significant difference in the number of pollens germinating in the control group, with no germination observed in most florets.

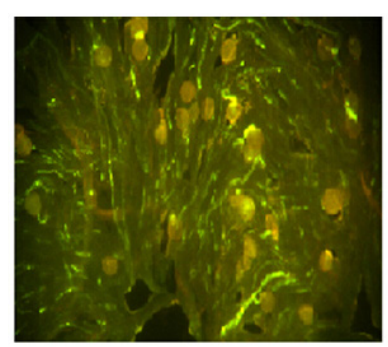

A

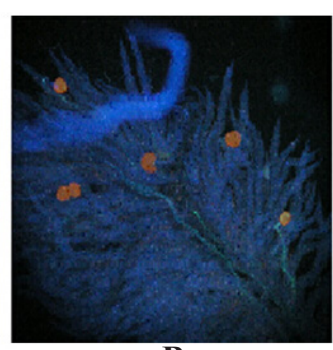

B

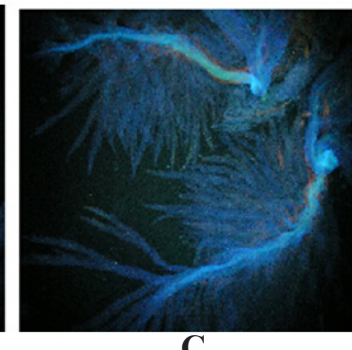

C

Figure 3. Pollen number and pollen germination on stigma. Stigma of plant with application of silicon. A. About $90 \%$ of the plants with pollen number greater than 25 per stigma. B. Stigma of plant with non-application of silicon. C. Approximatelly $17 \%$ of the plants with no pollen on the stigma. 


\section{Effect of silicon on the number, viability, and seed-setting rate of pollen}

In vitro germination culture was conducted using F1 pollen with and without silicon treatment for the 2 intersubspecies hybrids. The in vitro germination rate of pollen after silicon processing was $75.15 \pm 1.28 \%$ and $76.23 \pm 1.34 \%$ (Figure $4 \mathrm{~B}$ ), in treatment and non-treatment group, respectively, whereas the in vitro germination rate without silicon processing was 46.7 $\pm 1.5 \%$ and $48.13 \pm 1.49 \%$, in treatment and non-treatment group respectively (Figure $4 \mathrm{~A}$ ). A significant difference was observed between the 2 groups. Furthermore, pollen germination in vitro showed a more obvious group effect. Plants with a larger number of pollen grains germinated more easily. After silicon treatment of F1 of 'Taichung 65' x 'Guangluai 4' and 'Ludao' $\mathrm{x}$ 'Qiuguang', the seed-setting rates were $65.5 \pm 1.2 \%$ and $68.7 \pm 1.5 \%$, respectively. Thus, our findings demonstrated that the rate of anther dehiscence, the number of pollen per stigma of Fl plants, the potential of pollen grain germination, and the fertility of spikelets could be significantly improved by application of a silicon fertilizer.

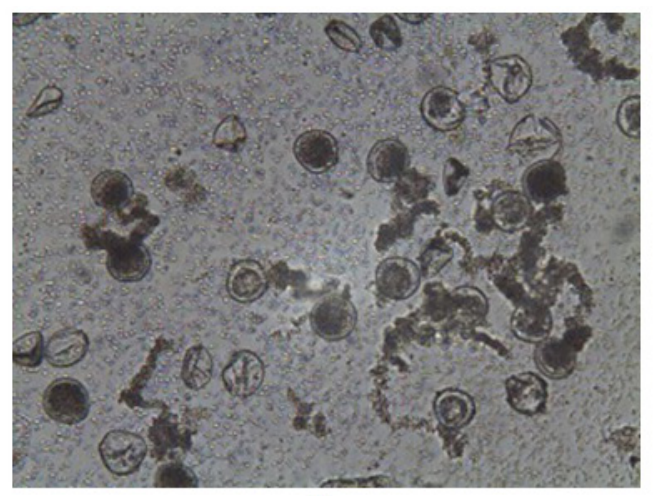

$\mathrm{A}$

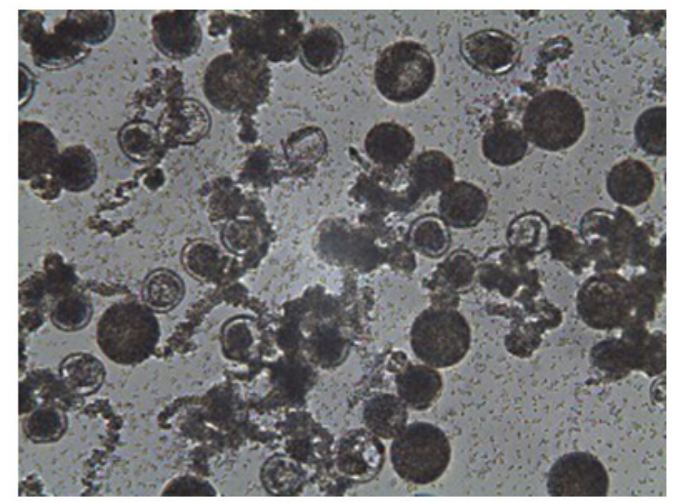

B

Figure 4. In vitro germination of rice pollen.

\section{DISCUSSION}

$\mathrm{Lu}$ rice is a unique rice germplasm with the weed habit in the downstream areas of the Yangtze River. It is mainly located in the Lianyungang, Jiangsu Province $\left(34^{\circ} 33^{\prime}\right.$ to $34^{\circ} 46^{\prime} \mathrm{N}$, $119^{\circ} 13^{\prime}$ to $\left.119^{\circ} 30^{\prime} \mathrm{E}\right)$. Its taxonomic status in the genus Oryza is controversial. In this study, by using Hybrids subspecies and Lu rice with subspecies japonica hybrids, these two types of sub-species hybrids were studied. We found that semi-sterility existed in both subspecies. The largest limitation to the effective use of subspecies resources are the semi-sterility in subspecies between hybrids. However, we also found that the fertility sac of the hybrids between the 2 subspecies hybrids was normal, with the male showing half-infertility. Theoretically, even if this type of sub-species hybrid accounted for only $10 \%$ of normal pollen and the stigma of all plants received sufficient amounts of pollen, fertilization could occur. The present study further analyzed these 2 types of subspecies hybrids.

Paddy rice is a typical siliconphile, with silica content in vivo up to 10 to $20 \%$, which is 10 -fold higher than that of nitrogen and 20 -fold, that of phosphorus. Silicon fertilizer shows 
positive effects on the growth and development of the crop, and the effect is more significant in gramineous crops. Numerous studies have indicated that after rational application of silicon fertilizer to rice, yield and resistance to lodging are very significantly increased (Ma et al., 1989; Epstein, 1999; Shang et al., 2009). Studies have shown that at high temperatures stress, silicon can promote the dehiscence rate of rice anther and the pollination of stigma (Li et al., 2005). Therefore, we examined whether increasing silicon would increase the seed-setting rate of the subspecies. The results showed that increased silicon not only promoted cracking of the rice anther but also increased the number of pollen on the stigma. Additionally, silicon increased the pollen germination rate, therefore improving the seed-setting rate of subspecies hybrids. Silicon has an important impact on the mechanical strength of the cell wall. Thus, we predict that the addition of silicon is likely to affect the mechanical strength and the thickness of the anther wall, affecting cracking of the anther and increase in the number of pollen grains on the stigma. The fertility sac of the hybrids between the 2 subspecies hybrids was normal, increasing the rate of seed setting.

\section{Conflicts of interest}

The authors declare no conflict of interest.

\section{ACKNOWLEDGMENTS}

Research supported by grants from the National Natural Science Foundation of China (\#1304317 and \#31370219) and the Ministry of Science and Technology of China (\#2013GA750005).

\section{REFERENCES}

Ekanayake IJ, Steponkus PL and De Datta SK (1990). Sensitivity of pollination to water deficits at anthesis in upland rice. Crop Sci. 30: 310-314.

Epstein E (1999). Silicon. Annu. Rev. Plant Physiol. Plant Mol. Biol. 50: 641-664.

Kitamura E (1962). Studies on cytoplasmic sterility of hybrids in distantly related varieties of rice, Oryza sativa L. I. Fertility of the $\mathrm{F}_{1}$ hybrids between strains derived from certain Philippine $\mathrm{x}$ Japanese variety crosses and Japanese varieties. Jpn. J. Breed. 12: 81-84.

Li WB, Wang H and Zhang FS (2005). Effects of silicon on anther dehiscence and pollen shedding in rice under high temperature stress. Acta Agron. Sin. 31: 134-136.

Li WC, Jiang L, Zhou SR, Wang CM, et al. (2007). Fine mapping of pss1, a pollen semi-sterile gene in rice (Oryza sativa L.). Theor. Appl. Genet. 114: 939-946.

Liu YS, Sun JS and Zhou KD (1997). Cytological basis causing spikelet sterility of intersubspecific hybrid in Oryza sativa. ABES 30: 355-359.

Ma JF, Nishimura K and Takahashi E (1989). Effect of silicon on the growth of rice plant at different growth stages. Soil Sci. Plant Nutr. 35: 347-356.

Matsui T, Omasa K and Horie T (2000). High Temperature at flowering inhibits swelling of pollen grains, a driving force of thecae dehiscence in rice (Oryza sativa L.). Plant Prod. Sci. 3: 430-434.

Shang QY, Zhang WZ, Han YD, et al. (2009). Effect of silicon fertilizer application on yield and grain quality of japonica rice from northeast China. Chin. J. Rice Sci. 23: 661-664.

Wang SH, Chen F and Zhou KD (2000). In vitro pollen germination of rice (Oryza sativa L.). Acta Agron. Sin. 26: 609-612. Yoshida S, Forno DA, Cook JH and Gomes KA (1976). Routine procedure for growing rice plaints in culture solution. In: Laboratory Manual for Physiological Studies of Rice. 3rd edn. The International Rice Research Institude. Los Banos, Laguna, Philippines, 61-65. 